\title{
Maximally discordant mixed states of two qubits
}

\author{
Fernando Galve, Gian Luca Giorgi, and Roberta Zambrini \\ IFISC (UIB-CSIC), Instituto de Física Interdisciplinar y Sistemas Complejos, UIB Campus, E-07122 Palma de Mallorca, Spain
}

(Received 13 July 2010; published 13 January 2011)

\begin{abstract}
We study the relative strength of classical and quantum correlations, as measured by discord, for two-qubit states. Quantum correlations appear only in the presence of classical correlations, while the reverse is not always true. We identify the family of states that maximize the discord for a given value of the classical correlations and show that the largest attainable discord for mixed states is greater than for pure states. The difference between discord and entanglement is emphasized by the remarkable fact that these states do not maximize entanglement and are, in some cases, even separable. Finally, by random generation of density matrices uniformly distributed over the whole Hilbert space, we quantify the frequency of the appearance of quantum and classical correlations for different ranks.
\end{abstract}

DOI: $10.1103 /$ PhysRevA.83.012102

PACS number(s): 03.65.Ud, 03.67.Mn, 03.65.Ta

\section{INTRODUCTION}

One of the most striking features of quantum mechanics is entanglement, first considered (although not by that name) by Einstein, Podolsky, and Rosen in their seminal paper in 1935 [1]. This is an exclusively quantum feature of composite states that can not be written as mixtures of product states. Theoretical and experimental research activity to characterize entanglement has been particularly intense in the last decade (see review [2] and references therein), being part of a broader endeavor to explore distinctive aspects of quantum versus classical physics and novel resources for quantum information purposes [3]. An important issue considered by several authors [4-6] is the existence of quantum correlations beyond entanglement in separable states. As a matter of fact, examples of improved quantum computing tasks not relying on entanglement have been reported [7].

\section{QUANTUM CORRELATIONS: THE DISCORD}

Two complementary approaches on quantum correlations are receiving great attention [4,5]. In Ref. [4], quantum correlations (quantum discord) have been associated to the difference of two classically equivalent expressions for the mutual information, $\mathcal{I}$ and $\mathcal{J}$. In particular, the quantum mutual information is defined as $\mathcal{I}(\varrho)=S\left(\varrho_{A}\right)+S\left(\varrho_{B}\right)-$ $S(\varrho)$, where $S$ stands for the von Neumann entropy and $\varrho_{A(B)}$ is the reduced density matrix of each subsystem. The classically equivalent expression stemming from Bayes rule is $\mathcal{J}(\varrho)_{\left\{\Pi_{j}^{B}\right\}}=S\left(\varrho_{A}\right)-S\left(A \mid\left\{\Pi_{j}^{B}\right\}\right)$, with the conditional entropy defined as $S\left(A \mid\left\{\Pi_{j}^{B}\right\}\right)=\sum_{i} p_{i} S\left(\varrho_{A \mid \Pi_{i}^{B}}\right), \quad p_{i}=\operatorname{Tr}_{A B}\left(\Pi_{i}^{B} \varrho\right)$, and where $\varrho_{A \mid \Pi_{i}^{B}}=\Pi_{i}^{B} \varrho \Pi_{i}^{B} / p_{i}$ is the density matrix after a complete projective measurement $\left(\left\{\Pi_{j}^{B}\right\}\right)$ has been performed on $B$. Quantum discord is obtained by minimizing the difference $\mathcal{I}(\varrho)-\mathcal{J}(\varrho)$ :

$$
\delta_{A: B}(\varrho)=\min _{\left\{\Pi_{i}^{B}\right\}}\left[S\left(\varrho_{B}\right)-S(\varrho)+S\left(A \mid\left\{\Pi_{i}^{B}\right\}\right)\right],
$$

that is, when measurement is performed in the basis that disturbs the state the least. A complementary approach was described in Ref. [5], defining classical correlations and showing that total correlations given by the mutual information are actually larger than the sum of the classical correlations and entanglement $E$ [8]. As a matter of fact, the quantum mutual information can be seen as the sum of quantum correlations [4] $\delta_{A: B}(\varrho)$ and the classical correlations [5] $\max _{\left\{\Pi_{i}^{B}\right\}} \mathcal{J}(\varrho)_{\left\{\Pi_{j}^{B}\right\}}$. We remark that, in [4], the discord is defined in terms of orthogonal (perfect) measurements. Even if possible generalizations to positive-operator-valued measurements (POVM) were considered at the end of that paper, as well as in [5], calculations of discord in the literature generally consider only orthogonal measurements (see, e.g., [4,7,9-11,16,17]).

In contrast with state separability, this new paradigm of quantumness of correlations is measurement oriented, considering an experiment where all information of a system $A$ is extracted by measuring another system $B$. According to this measure, a state is classically correlated only when consecutive measurements of system $B$ yield the same picture of the state of system $A$, which is achieved after decoherence into the pointer basis of $B[4,9]$. For pure states, quantum discord is equivalent to entanglement and actually has the same value of classical correlations [5]. On the other hand, when mixed states are considered, entanglement does significantly depart from the quantum discord, the difference being positive for some states and negative for others [10]. As the definition of discord comes from a minimization over all possible measurement bases, only a few general results have been reported. Analytic expressions are known for states of two qubits with maximally mixed marginals [10] for $X$-shaped states [11] and also for Gaussian states of continuous-variable systems [12].

In this paper, we explore the whole Hilbert space of two qubits to gain insight on their correlations for mixed states of different ranks. Our main goal is to discern the proportion of quantum to classical correlations between the two qubits. We find the most nonclassical two-qubit states, i.e., the family with maximal quantum discord versus classical correlations, were formed by mixed states of rank 2 and 3, which we name maximally discordant mixed states (MDMS). The analogous effort to identify largest deviations from classical states has led to mixed states maximizing entanglement versus purity [13]. In contrast with maximally entangled mixed states (MEMS), where a geometrical property such as separability could be considered with several constraints, the MDMS are naturally defined and allow us to quantify the relative strength of quantum and classical correlations, which are related in a 
closed from. The MDMS proposed here do not maximize entanglement for a given amount of classical correlations; part of them are, in fact, separable. This pinpoints the fundamental difference between entanglement and discord for mixed states, in opposition to their exact equivalence for pure states. Their discord also implies that quantum correlations are always accompanied by classical correlations, while the reverse is not always true. Furthermore, we study the probability of states with a given amount of discord in the whole two-qubit Hilbert space, supporting the recent result that the closed set of purely classically correlated states $\left(\delta_{A: B}=0\right)$ has a measure of zero [14], and we compare it with the probability for classical correlations and entanglement. Only such states with no discord have been shown to ensure a future non-negative evolution in the presence of dissipation [15], while discord can not be made zero in a finite time by any Markovian map [14]. Furthermore, even in the presence of a noisy environment, for some family of initial states, discord can be robust under decoherence for a finite time [16]. Experimental results with polarization entangled photons also have been reported recently [17].

\section{MIXED STATES WITH THE LARGEST DISCORD}

As pure states with maximum entanglement that are also maximally discordant, Bell states are a natural starting point to identify states with large discord; thus, by mixing these states with other components, it is quite plausible that we find states with a large proportion of quantum versus classical correlations. We then consider an example of a mixture of any Bell state $|\psi\rangle$ with another orthogonal pure state, i.e., $\varrho=\epsilon|\psi\rangle\langle\psi|+(1-\epsilon)| \phi\rangle\langle\phi|$. If $|\phi\rangle$ is any other Bell state, then $\delta_{A: B}=E$ (with $E$ being entanglement) and $\mathcal{J}=1$, and a worse discord is found than in that of pure states with the same classical correlation $\mathcal{J}$. In contrast, a mixture of a Bell state and a state of the computational basis of the opposite parity sector gives a huge amount of discord. We thus consider states

$$
\varrho=\epsilon\left|\Phi^{+}\right\rangle\left\langle\Phi^{+}|+(1-\epsilon)| 01\right\rangle\langle 01|,
$$

with $\left|\Phi^{+}\right\rangle=(|00\rangle+|11\rangle) / \sqrt{2}$ as usual. With local unitary operations, which leave discord invariant, we can obtain from this ansatz any combination of a Bell state mixed with a computational basis state of opposite parity (number of 1's in the state). The expression of discord for states (2) is invariant under permutation of the individual labels $A \leftrightarrow B$. As a matter of fact, we find that states (2) maximize the symmetrized version of discord $\left(\delta_{A: B}+\delta_{B: A}\right) / 2$ for all rank-2 matrices.

It can be shown that, when we compare with a numerical scan of Hilbert's space, this state is too symmetric. In fact, a better option in terms of discord is obtained if some amount of entanglement is sacrificed for the good of quantum correlations. This results from asymmetrizing the maximally entangled (Bell) state leading to the ansatz

$$
\varrho^{(\mathrm{R} 2)}=\epsilon\left|\tilde{\Phi}^{+}\right\rangle\left\langle\tilde{\Phi}^{+}|+(1-\epsilon)| 01\right\rangle\langle 01|,
$$

with $\left.\left|\tilde{\Phi}^{+}\right\rangle=\sqrt{p}|00\rangle+\sqrt{1-p}|11\rangle\right)$, which coincides with a Bell state for $p=1 / 2$. The increase of discord for the states (3) with respect to (2) highlights the importance of the

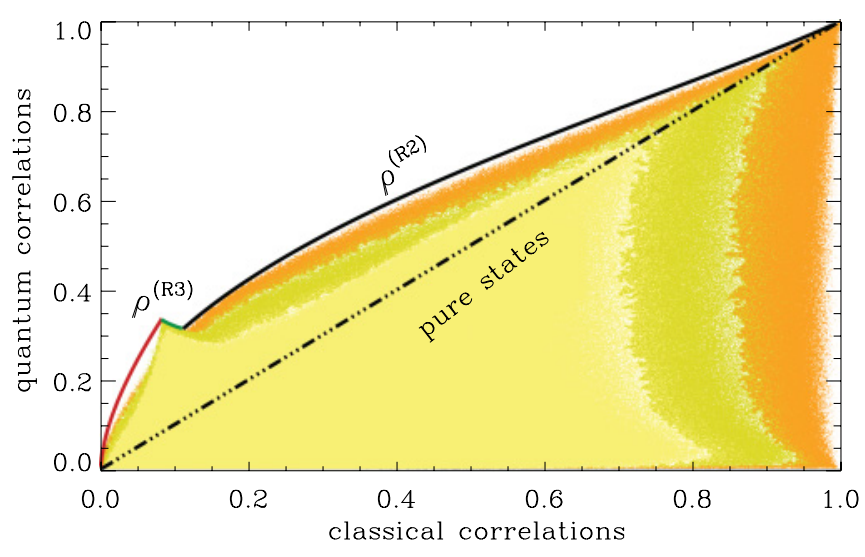

FIG. 1. (Color online) Quantum discord $\left(\delta_{A: B}\right)$ vs classical correlations $(\mathcal{J})$ for two-qubit states. The MDMS family (continuous line) gives two segments for $\operatorname{rank} 3\left(\varrho^{(\mathrm{R} 3)}\right)$ and one for rank 2 $\left(\varrho^{(\mathrm{R} 2)}\right)$. Layers of $10^{8}$ random matrices of rank 2 (dark points), 3 (intermediate color), and 4 (lighter color) are superimposed. For pure states (dotted-dashed line), $\delta_{A: B}=\mathcal{J}=E$.

asymmetric definition of quantum discord based on the asymmetric operation of measuring $B$ in order to know about $A$. The discord for this family can be written once we know the conditional entropies $\min _{\left\{\Pi_{i}^{B}\right\}} S\left(A \mid\left\{\Pi_{i}^{B}\right\}\right)=\left(x \log _{2} \frac{1-x}{1+x}-\right.$ $\left.\log _{2} y\right) / 2$, with $x=\sqrt{1-4 y}$ and $y=\epsilon(1-p)(1-\epsilon)$, while for $\delta_{B: A}$, we need to use $y=\epsilon p(1-\epsilon)$.The total and reduced entropies are easy to calculate once we notice that the ansatz is given in spectral decomposition, although we do not give the whole expression for reasons of space.

The family of MDMS is obtained for an optimal function $\epsilon_{\mathrm{opt}}(p)$ through the use of Lagrange multipliers, as detailed later in this paper. Once this optimal curve $\epsilon_{\mathrm{opt}}(p)$ is used, Eq. (3) gives the family of states that maximize the quantum part of correlations for a given classical part, when all rank-2 (R2) states are considered. As shown in Fig. 1, these $\varrho^{(\mathrm{R} 2)}$ states are the MDMS for a large range of classical correlations.

In order to find the states that maximize $\delta_{A: B}$, we also need to use rank-3 states. In this case, it can be checked that asymmetrization of the Bell-state component $|\phi\rangle$ does not help, and that the best choice for an ansatz is

$\varrho^{(\mathrm{R} 3)}=\epsilon\left|\Phi^{+}\right\rangle\left\langle\Phi^{+}\right|+(1-\epsilon)[m|01\rangle\langle 01|+(1-m)| 10\rangle\langle 10|]$.

As before, any combination of Bell state plus two components of opposite parity belonging to the computational basis will do. The optimal $\epsilon_{\mathrm{opt}}(m)$ is discussed later and leads to the family of states $\varrho^{(\mathrm{R} 3)}$ maximizing discord for small classical correlations. This optimal family has the property of being separable (not entangled), as shown in Fig. 2, while it maximizes quantum discord, highlighting the inequivalence of these measures of quantumness. It is actually found that, for these states, the discord amounts to the weight of the Bell component, and the simple relation $\delta_{A: B}=\delta_{B: A}=\epsilon$ holds. For completeness, the entanglement (as quantified by the concurrence [8]) yields $E\left(\varrho^{(\mathrm{R} 2)}\right)=2 \epsilon \sqrt{p(1-p)}$ and $E\left(\varrho^{(\mathrm{R} 3)}\right)=$ $\max [0, \epsilon-2(1-\epsilon) \sqrt{m(1-m)}]$. Although MDMS of rank 3 are separable, the MDMS of rank 2 have a high amount of entanglement, even if they do not maximize it (Fig. 2) As 


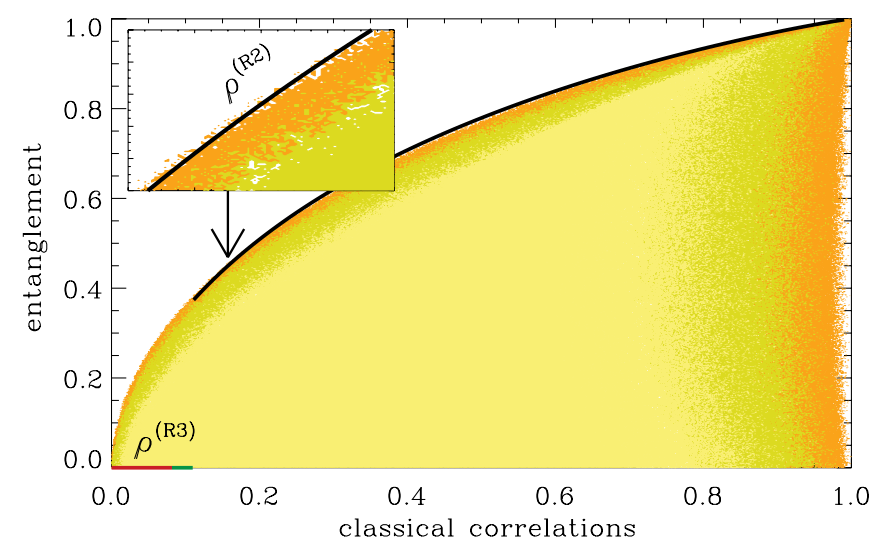

FIG. 2. (Color online) Entanglement ( $E$ ) vs classical correlations $(\mathcal{J})$. Random matrices and lines for the MDMS family as in Fig. 1. MDMS of rank 3 are separable, while $\varrho^{(\mathrm{R} 2)}$ states show large entanglement, even if not maximum, as seen in the inset.

mentioned before, asymmetrization of the Bell component increases quantum correlations at the expense of entanglement.

An intriguing state is that of the singular point for $\varrho^{(\mathrm{R} 3)}$ shown in Fig. 1: $\varrho^{\text {cusp }}=\left(\left|\Phi^{+}\right\rangle\left\langle\Phi^{+}|+| 01\right\rangle\langle 01|+| 10\rangle\langle 10|\right) / 3$ reaches the lowest possible purity for a rank-3 state and is separable, yet with a high level of discord. Another important feature emerging from Fig. 1 is that MDMS have a discord larger than pure states $\left(\delta_{A: B}>\mathcal{J}\right)$ and satisfy $\mathcal{J}=0$ only when $\delta_{A: B}=0$, thus showing the lack of states with finite quantum without classical correlations. In other words, no state of two qubits is purely quantum. Maybe less surprisingly, there are no states with finite entanglement and zero classical correlations (Fig. 2).

\section{DETAILED ANALYSIS}

We first consider the commonly accepted definition of discord, obtained expressing the measurement projectors as $\Pi_{j}^{B}=\left|\psi_{j}^{B}\right\rangle\left\langle\psi_{j}^{B}\right|, j=1,2$, with $\left|\psi_{1}^{B}\right\rangle=\cos \theta|0\rangle+$ $e^{i \phi} \sin \theta|1\rangle$ and $\left|\psi_{2}^{B}\right\rangle=-e^{-i \phi} \sin \theta|0\rangle+\cos \theta|1\rangle$ [4]. The angles minimizing Eq. (1) for the states (3), and thus giving the correct discord, are $\theta=\pi / 4+n \pi / 2$ and any value of $\phi$. We then can choose $\phi=0$ and hence the optimal projectors are $\Pi_{1}^{B}=\left|+{ }^{B}\right\rangle\left\langle+{ }^{B}\right|$ and $\Pi_{2}^{B}=\left|{ }^{B}\right\rangle\left\langle-{ }^{B}\right|$, with $| \pm\rangle=(|0\rangle \pm|1\rangle) / \sqrt{2}$.

The method of Lagrange multipliers allows us to maximize first the discord for the rank-2 family $\delta_{A: B}\left(\varrho^{(\mathrm{R} 2)}\right) \equiv \delta(\epsilon, p)$ while keeping its classical correlations $\mathcal{J}\left(\varrho^{(\mathrm{R} 2)}\right) \equiv \mathcal{J}_{0}(\epsilon, p)$ constant (and the same for the rank-3 family $\varrho^{(\mathrm{R} 3)}$ ). This is achieved through definition of the function $\Lambda(\epsilon, p, \lambda)=$ $\delta(\epsilon, p)+\lambda\left[\mathcal{J}(\epsilon, p)-\mathcal{J}_{0}\right]$, where $\lambda$ is the Lagrange multiplier and $\mathcal{J}_{0}$ is an arbitrary but fixed amount of classical correlation. The extremization procedure is then simply the simultaneous solution of the three equations $\partial_{\mu} \Lambda=0$, with $\mu=\lambda, \epsilon, p$. From the first equation, $\mathcal{J}(\epsilon, p)=\mathcal{J}_{0}$ is obtained, as expected. From the last two equations, we can isolate $\lambda$ yielding the extremality condition

$$
\partial_{\epsilon} \delta / \partial_{\epsilon} \mathcal{J}=\partial_{p} \delta / \partial_{p} \mathcal{J}
$$

We stress that this condition is equivalent to maximization of $\delta$ versus $\mathcal{I}$, or minimization of $\mathcal{J}$ with respect to $\mathcal{I}$, due to the closed relation $\mathcal{I}=\delta+\mathcal{J}$. These quantities present nontrivial trigonometric relations, leading to a transcendental equation, the solution of which can only be given numerically.

The same procedure is followed for the rank-3 family $\varrho^{(\mathrm{R} 3)}$, with $m$ playing the role of $p$. In this case, obtaining $\epsilon_{\text {opt }}(m)$ is a bit trickier, due to the fact that there are two optimal angles (each of them good for different ranges of $\epsilon$ and $m) \theta=0, \pi / 4$; the angle $\phi$ is again not important. We can consider for the moment that projector maximization of discord has been simplified to $\delta_{A: B}\left(\varrho^{(\mathrm{R} 3)}\right)=\min \left(\delta_{0}, \delta_{\pi / 4}\right)$, the latter being functions of $\epsilon$ and $m$. The goal is to find the zero(es) of the function $\partial_{\epsilon} \delta / \partial_{\epsilon} \mathcal{J}-\partial_{m} \delta / \partial_{m} \mathcal{J}$, which of course needs the knowledge of when to use one angle or the other. However, the problem is greatly reduced by noticing that the latter function is positive when using $\delta_{0}$ and negative when using $\delta_{\pi / 4}$. This means that the zero of such function occurs exactly (and conveniently) when $\delta_{0}(\epsilon, m)=\delta_{\pi / 4}(\epsilon, m)$. Again, the solution to this transcendental equation can only be given numerically.

Finally, the MDMS are a family of states

$$
\begin{aligned}
\varrho^{\mathrm{MDMS}}= & \epsilon\left|\tilde{\Phi}^{+}\right\rangle\left\langle\tilde{\Phi}^{+}\right|+(1-\epsilon)[m|01\rangle\langle 01| \\
& +(1-m)|10\rangle\langle 10|],
\end{aligned}
$$

where the optimum choice of parameters gives the three curves in Fig. 1, two of them rank 3 and the other rank 2. The first curve, going from zero discord up to the cusp, is the rank-3 family $\varrho^{(\mathrm{R} 3)}$ with $\epsilon_{\mathrm{opt}}(m)$ given by the solution of $\delta_{0}=\delta_{\pi / 4}$. It is restricted to the domain $m \in[0,1], \epsilon \in[0,1 / 3]$. The second branch of MDMS is given by $\varrho^{(\mathrm{R} 3)}$ with $m=$ $1 / 2$ with domain $\epsilon \in[1 / 3,0.385]$, approximately. These two curves correspond to separable states, as noted in Fig. 2. The remaining curve of MDMS is the rank-2 family $\varrho^{(\mathrm{R} 2)}$ when the optimal function $\epsilon_{\mathrm{opt}}(p)$ given by Eq. (5) is used, and for $\epsilon$ approximately in the interval $[0.408,1]$. One might wonder how the picture changes if more general (nonorthogonal) measurements are considered. It has been shown that, for two qubits, discord is extremized exclusively by rank-1 POVMs with a maximum of four elements [21,22]. Perfect orthogonal measurements correspond to the case with the two elements considered above. Considering POVMs with measurement operators $E_{i}$, the measured density matrix takes the form $\varrho_{A \mid E_{i}}=E_{i} \varrho / p_{i}$ with probability $p_{i}=\operatorname{Tr}_{A B}\left(E_{i} \varrho\right)$. Even using the general measurement given by POVMs of four elements, we find the same discord for the MDMS, meaning that they represent the absolute border of maximally nonclassically correlated states of two qubits. A detailed analysis about the full Hilbert space will be presented elsewhere [23].

\section{STATISTICS}

Since our random generation of density matrices is uniform in the Hilbert space, preserving the Haar measure [18,19], we can measure the frequency of the appearance of states with different properties, as shown in Fig. 3, for different ranks. Some main features arise for all quantities investigated: (i) Zero correlations (be they quantum, classical, or mutual information) have zero probability. Notably, entanglement is the exception (consistently with Ref. [19]), where only rank-2 states have a probability zero of being separable. (ii) The lower 


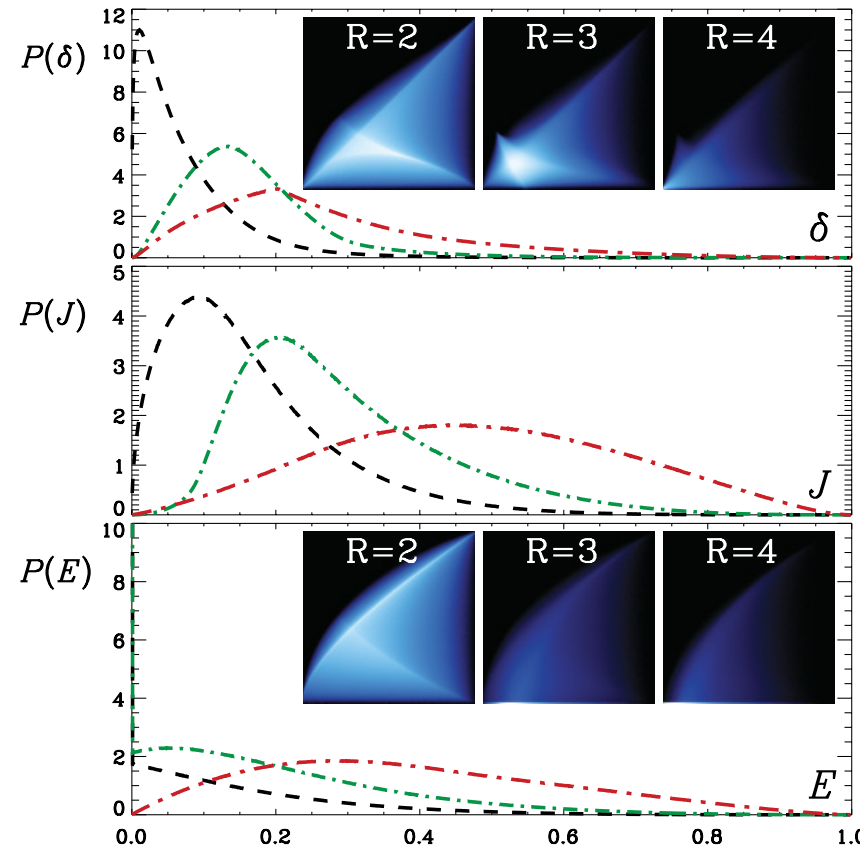

FIG. 3. (Color online) Probability (density) to find a two-qubit state with a given amount of quantum discord $\delta$, classical correlations $\mathcal{J}$, and entanglement $E$, respectively, for ranks 2 (dashed), 3 (dotdashed), and 4 (dot-dot-dashed). The insets show these probabilities (larger for light color) for the quantity under study ( $y$ axis) against classical correlations $\mathcal{J}$ ( $x$ axis) for different ranks.

the rank, the higher the typical amount of correlations. This is quite understandable, since higher ranks describe more mixed states. (iii) It is more probable to find states with more abundance of classical rather than quantum correlations. We note that only $\sim 7.45 \%$ of the two-qubit states has greater discord than classical correlations. If restricted to lower ranks, we observe that rank- 2 matrices yield $\sim 10.76 \%$, and rank-3 matrices yield $\sim 16.3 \%$. Finally, as shown in the insets in Fig. 3 , the border of MDMS seems to be rather improbable to find in the space of two-qubit states, except for the middle branch in the cusp, meaning that such extremely nonclassical states are quite rare.

\section{CONCLUSIONS}

The unique family of two-qubit mixed states with maximal proportion of quantum discord versus classical correlations [the MDMS in Eq. (6)] has been identified. Part of them have rank 2 and are highly, although not maximally, entangled, while the other part has rank 3 and is separable, thus providing another evidence of the inequivalence of these two measures of quantumness. We have shown that the presence of discord is a sufficient but not necessary condition to have nonvanishing classical correlations. The uniform generation of states (random states preserving Haar measure) allowed us to find the probabilities and typical values of classical and quantum correlations, as well as entanglement. We verified that completely (either quantum or classical) uncorrelated states are very rare, as well as extreme nonclassical states. The identification of MDMS, together with the ability to experimentally generate [20] and characterize [17] these states, is a key tool to establish the fundamental difference in performance of quantum versus classical information [7].

\section{ACKNOWLEDGMENT}

Funding from FISICOS (Project No. FIS2007-60327), ECuSCo (Project No. 200850I047), and CoQuSys (Project No. 200450E566) and the Juan de la Cierva program are acknowledged.
[1] A. Einstein, B. Podolsky, and N. Rosen, Phys. Rev. 47, 777 (1935).

[2] R. Horodecki, P. Horodecki, M. Horodecki, and K. Horodecki, Rev. Mod. Phys. 81, 865 (2009).

[3] M. A. Nielsen and I. L. Chuang, Quantum Computation and Quantum Information (Cambridge University Press, Cambridge, UK, 2000).

[4] H. Ollivier and W. H. Zurek, Phys. Rev. Lett. 88, 017901 (2001).

[5] L. Henderson and V. Vedral, J. Phys. A 34, 6899 (2001).

[6] M. Horodecki, K. Horodecki, P. Horodecki, R. Horodecki, J. Oppenheim, A. Sen(De), and U. Sen, Phys. Rev. Lett. 90, 100402 (2003).

[7] E. Knill and R. Laflamme, Phys. Rev. Lett. 81, 5672 (1998); S. L. Braunstein, C. M. Caves, R. Jozsa, N. Linden, S. Popescu, and R. Schack, ibid. 83, 1054 (1999); D. A. Meyer, ibid. 85, 2014 (2000); E. Biham, G. Brassard, D. Kenigsberg, and T. Mor, Theor. Comput. Sci. 320, 15 (2004); A. Datta, A. Shaji, and C. M. Caves, Phys. Rev. Lett. 100, 050502 (2008); B. P. Lanyon, M. Barbieri, M. P. Almeida, and A. G. White, ibid. 101, 200501 (2008); V. Vedral, Found. Phys. 40, 1141 (2010).
[8] W. K. Wootters, Phys. Rev. Lett. 80, 2245 (1998).

[9] J. Maziero, L. C. Cèleri, R. M. Serra, and V. Vedral, Phys. Rev. A 80, 044102 (2009).

[10] S. Luo, Phys. Rev. A 77, 042303 (2008).

[11] M. Ali, A. R. P. Rau, and G. Alber, Phys. Rev. A 81, 042105 (2010).

[12] P. Giorda and M. G. A. Paris, Phys. Rev. Lett. 105, 020503 (2010); G. Adesso and A. Datta, ibid. 105, 030501 (2010).

[13] S. Ishizaka and T. Hiroshima, Phys. Rev. A 62, 022310 (2000); F. Verstraete, K. Audenaert, and B. De Moor, ibid. 64, 012316 (2001).

[14] A. Ferraro, L. Aolita, D. Cavalcanti, F. M. Cucchietti, and A. Acín, Phys. Rev. A 81, 052318 (2010).

[15] C. A. Rodríguez-Rosario, K. Modi, A. Kuah, A. Shaji, and E. C. G. Sudarshan, J. Phys. A 41, 205301 (2008); A. Shabani and D. A. Lidar, Phys. Rev. Lett. 102, 100402 (2009).

[16] L. Mazzola, J. Piilo, and S. Maniscalco, Phys. Rev. Lett. 104, 200401 (2010).

[17] J.-S. Xu, X.-Y. Xu, C.-F. Li, C.-J. Zhang, X.-B. Zou, and G.-C. Guo, Nature Commun. 1, 7 (2010).

[18] K. Zyczkowski and M. Kus, J. Phys. A 27, 4235 (1994). 
[19] K. Zyczkowski, P. Horodecki, A. Sanpera, and M. Lewenstein, Phys. Rev. A 58, 883 (1998).

[20] F. F. Fanchini, L. K. Castelano, and A. O. Caldeira, New J. Phys. 12, 073009 (2010).
[21] A. Datta, eprint arXiv:0807.4490.

[22] G. M. D'Ariano, P. Perinotti, and P. Lo Presti, J. Phys. A 38, 5979 (2005).

[23] F. Galve, G. L. Giorgi, and R. Zambrini (unpublished). 